\title{
CÁlCiO E VITAMINA D EM ADULTOS ATENDIDOS EM AMBULATÓRIO DE NUTRIÇÃO
}

\section{Calcium and vitamin $D$ in adults attending an outpatient nutrition clinic}

\section{Calcio y vitamina D en adultos asistidos en ambulatorio de nutrición}

\author{
Bruna Amanda Martini \\ Centro Universitário UNIVATES - Lajeado - RS - Brasil \\ Simara Rufatto Conde \\ Centro Universitário UNIVATES - Lajeado - RS - Brasil
}

\section{Fernanda Scherer Adami}

Centro Universitário UNIVATES - Lajeado - RS - Brasil

\section{Patrícia Fassina}

Centro Universitário UNIVATES - Lajeado - RS - Brasil

\section{RESUMO}

Objetivo: Avaliar o consumo de cálcio e vitamina D de pacientes atendidos em um ambulatório de Nutrição de referência. Métodos: Estudo transversal quantitativo realizado no período de novembro de 2015 a junho de 2016 avaliou 1.000 recordatórios alimentares de pacientes na faixa etária de 20 a 59 anos do ambulatório de Nutrição de um centro universitário do interior do Rio Grande do Sul, Brasil. Excluíram-se os recordatórios com medidas caseiras incompletas. Para a padronização dessas medidas utilizou-se registro fotográfico de um livro de medidas caseiras. Utilizou-se como referência para análise de ingestão do cálcio e vitamina D a Dietary Reference Intakes. Os dados foram analisados por meio de estatística descritiva e inferencial com nível de significância de 5\%. Resultados: O consumo de cálcio e de vitamina $\mathrm{D}$ foi simétrico, apresentando 89,5\% ( $\mathrm{n}=895)$ de inadequação do consumo de vitamina $\mathrm{D}$ e $86,6 \%$ (n=866) de inadequação do consumo de cálcio, portanto, abaixo da recomendação diária. Homens apresentaram ingestão significativamente maior de cálcio em comparação às mulheres $(p<0,001)$, enquanto o consumo de vitamina $\mathrm{D}$ não apresentou diferença significativa entre os sexos ( $\mathrm{p}=0,307)$. Conclusão: Verificou-se elevada inadequação quanto à ingestão de cálcio e vitamina D pela população estudada.

Descritores: Cálcio na Dieta; Vitamina D; Adulto.

\section{ABSTRACT}

Objective: To assess calcium and vitamin D intake in patients attending a reference outpatient nutrition clinic. Methods: Quantitative crosssectional study carried out from November 2015 to June 2016 to analyze 1,000 dietary recalls of patients aged 20 to 59 years attending an outpatient nutrition clinic of a university center in the countryside of Rio Grande do Sul. Recalls with incomplete household measures were excluded. Measures were standardized using pictures from a household measures book. The analysis of calcium and vitamin D intake was based on the Dietary Reference Intakes. Data were analyzed using descriptive and inferential statistics with a significance level of $5 \%$. Results: Calcium and vitamin D intake was symmetrical, with $89.5 \%(n=895)$ of inadequate vitamin $D$ intake and $86.6 \%(n=866)$ of inadequate calcium intake, i.e., below the daily recommendation. Men presented significantly higher calcium intake compared to women $(p<0.001)$, while vitamin D intake showed no significant differences between genders $(p=0.307)$. Conclusion: There was a high inadequacy of calcium and vitamin D intake in the population analyzed.

Descriptors: Calcium, Dietary; Vitamin D; Adult. 


\section{RESUMEN}

Objetivo: Evaluar el consumo de calcio y vitamina D de pacientes asistidos en un ambulatorio de nutrición de referencia. Métodos: Estudio transversal cuantitativo realizado en el periodo entre noviembre de 2015 y junio de 2016 evaluó 1.000 recordatorios alimentarios de pacientes en la franja de edad entre 20 y 59 años del ambulatorio de nutrición de un centro universitario de un pueblo de Rio Grande do Sul, Brasil. Se excluyeron los recordatorios con las medidas caseras incompletas. Para la estandarización de esas medidas se utilizó el registro fotográfico de un libro de medidas caseras. Se utilizó como referencia para el análisis de la ingesta de calcio y vitamina D la Dietary Reference Intakes. Los datos fueron analizados a través de la estadistica descriptiva e inferencial con el nivel de significación del 5\%. Resultados: El consumo de calcio y vitamina $D$ ha sido simétrico presentando el 89,5\% (n=895) de inadecuación para el consumo de vitamina $D$ y el 86,6\% $(n=866)$ de inadecuación para el consumo de calcio, por lo tanto, abajo de la recomendación diaria. Los hombres presentaron ingesta significativamente mayor de calcio en comparación con las mujeres $(p<0,001)$ mientras el consumo de vitamina $D$ no ha presentado diferencia significativa entre los sexos $(p=0,307)$. Conclusión: Se verificó una elevada inadecuación de la ingesta de calcio y vitamina $D$ de parte de la población estudiada.

Descriptores: Calcio en la Dieta; Vitamina D; Adulto.

\section{INTRODUÇÃO}

Para a promoção e manutenção da saúde, torna-se fundamental que se tenha uma alimentação adequada ${ }^{(1)}$. Para que se consiga uma boa saúde, e consequentemente uma boa saúde óssea, o melhor caminho é uma alimentação diversificada, principalmente, com os níveis corretos de cálcio e vitamina $\mathrm{D}^{(2)}$, os quais são fundamentais na manutenção da saúde óssea e redução do risco de osteoporose ${ }^{(3)}$.

Mesmo que o ser humano já tenha concluído a sua fase de crescimento, ele necessitará de uma dieta rica em nutrientes, principalmente cálcio, pois, perde-se diariamente uma considerável quantidade desse mineral ${ }^{(2)}$ A vitamina $\mathrm{D}$, juntamente com o cálcio possui como função manter a homeostase e intensificar o transporte de cálcio no intestino delgado, os quais aumentam sua absorção ${ }^{(4)}$.

A vitamina D (calciferol) é encontrada na forma animal: (vitamina D3 ou colecalciferol); ou vegetal: (vitamina D2 ou ergocalciferol); podendo ser ingerida por meio da alimentação ou formada em tecidos cutâneos endogenamente através da exposição solar ${ }^{(4,5)}$.

A necessidade da vitamina $\mathrm{D}$ varia conforme a faixa etária, permanecendo em $15 \mu \mathrm{g}$ por dia para ambos os sexos de $20 \mathrm{a}$ 59 anos $^{(6)}$, entretanto, poucos alimentos a possuem naturalmente ${ }^{(4,7)}$. Assim, sem o consumo diário de alimentos naturalmente ricos ou enriquecidos, os indivíduos podem acabar desenvolvendo deficiência nos níveis séricos de vitamina $\mathrm{D}$, principalmente em condições como: ausência de exposição diária à luz solar, uso de filtros solares, sedentarismo, estado nutricional, abuso de medicamentos orais que interferem na sua absorção, e dieta desequilibrada, levando à inadequação da ingestão da mesma ${ }^{(8,9)}$.

O cálcio é o mineral mais abundante do corpo humano, um nutriente de extrema importância para a realização de várias funções vitais do organismo, apresentando-se nos ossos (99\%), no plasma sanguíneo e em diversos tecidos (1\%), sendo o esqueleto seu principal reservatório ${ }^{(10)}$ Sua absorção intestinal é regulada pela vitamina $\mathrm{D}^{(11)}$, sendo afetado por fatores que influenciam negativamente sua biodisponibilidade e digestibilidade ${ }^{(10,12)}$.

A recomendação diária de cálcio, para ambos os sexos, de 20 a 59 anos é de $1.000 \mathrm{mg}$, sendo de $1.200 \mathrm{mg}$ para o sexo feminino a partir dos 50 anos de idade ${ }^{(13)}$. O consumo diário em excesso dos micronutrientes cálcio e vitamina $\mathrm{D}$, em quantidades superiores a $2.000 \mathrm{mg}$ e $4.000 \mathrm{UI}$, respectivamente, está relacionado a complicações, como a hipercalcemia ${ }^{(5)}$.

O cálcio é encontrado em alimentos, sendo eles sua melhor fonte. Os alimentos que possuem maior biodisponibilidade desse mineral são leites e seus derivados, certos peixes como sardinha, salmão e alguns folhosos verde-escuros ${ }^{(4)}$. A vitamina D não está muito disponível nos alimentos, sendo sua maior fonte em produtos animais do que em frutas, vegetais e cereais, sendo eles: óleo de fígado de peixe, peixes com alto teor de gordura, gema e produtos enriquecidos como: margarina fortificada, cereais e sucos ${ }^{(14)}$. Concentrações mais baixas de vitamina D estão associadas com elevados níveis de paratormônio (PTH) e consequentemente, altas taxas de reabsorção, e elevado risco de fraturas ${ }^{(7)}$.

O presente estudo se justifica pela necessidade de se conhecer a ingestão de cálcio e vitamina $D$ de uma população atendida em um ambulatório de nutrição de um centro universitário do interior do Rio Grande do Sul, para aprimorar as orientações voltadas à prevenção das complicações que podem ocorrer pela deficiência e/ou excesso da ingestão desses micronutrientes, assim dando ênfase para a promoção da saúde na população adulta a ser analisada.

Dessa forma, o objetivo deste estudo foi avaliar o consumo de cálcio e vitamina D de pacientes atendidos em um ambulatório de Nutrição de referência. 


\section{MÉTODOS}

Estudo transversal quantitativo realizado no período de novembro de 2015 a junho de 2016, no qual foram avaliados 1.000 recordatórios alimentares de usuários atendidos no ambulatório de nutrição de um centro universitário do interior do Rio Grande do Sul, Brasil.

O estudo incluiu os recordatórios preenchidos no período de 2010 a 2016 da totalidade dos adultos atendidos com idades entre 20 e 59 anos, de ambos os sexos sendo excluídos os recordatórios com registros incompletos e os indivíduos fora da faixa etária estipulada.

O consumo alimentar foi estimado por meio do recordatório alimentar de 24 horas registrado na anamnese alimentar, a partir do relato do paciente, durante os atendimentos realizados entre 2010 e 2016 por estagiários do curso de Nutrição, treinados e supervisionados por docente nutricionista. Para a padronização das medidas caseiras, durante a aplicação do recordatório, utilizou-se o registro fotográfico de um livro de medidas caseiras, intitulado "Métodos práticos para cálculos de dietas"(15) e utensílios para a melhor visualização e exatidão do paciente na hora do relato.

Os dados das medidas caseiras foram calculados com o auxílio do Software DietWin ${ }^{\circledR}$ Profissional 2008. Posteriormente, quantidades ingeridas de cálcio e vitamina D foram comparadas com a Dietary Reference Intakes (DRIs) para faixa etária e sexo $^{(13)}$, pois elas representam um novo paradigma para estabelecimento de indicadores nutricionais de consumo.

Para a obtenção dos resultados, calcularam-se estatísticas descritivas tais como: média, desvio padrão, mediana e amplitude interquartil para as variáveis quantitativas e frequência absoluta e relativa para as variáveis qualitativas. A normalidade das variáveis quantitativas foi avaliada pelo teste de Kolgomarov-Smirnoff, sendo a ingestão de cálcio e vitamina D variáveis com distribuição simétrica.

Para a comparação das variáveis entre os sexos, aplicou-se o teste t de Student nas amostras independentes, e, para as variáveis qualitativas, o teste Qui-quadrado. Os participantes foram divididos em grupos de acordo com a faixa etária $(<30$ anos, 31-40 anos, 41-50 anos, $>50$ anos). Para a comparação da ingestão de cálcio e vitamina D de acordo com a faixa etária, utilizou-se o teste Anova seguido pelo teste de Tukey para as análises post-hoc. Para a adequação de cálcio e vitamina D em relação à recomendação das DRIs, calculou-se a média de consumo do nutriente pelos participantes do estudo, dividido pelo valor recomendado, sendo o resultado multiplicado por 100 para ser expresso em \%, considerando-se significativos valores de $\mathrm{p}<0,05$. As análises estatísticas ocorreram no pacote estatístico Statistical Package for the Social Sciences (SPSS) 20.0.

O presente estudo recebeu aprovação pelo Comitê de Ética em Pesquisa do Centro Universitário Univates (COEP) sob Protocolo número 0087/10.

\section{RESULTADOS}

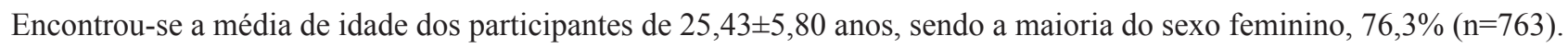

O consumo de cálcio e vitamina $\mathrm{D}$ entre os participantes do estudo foi simétrico, sendo a média de consumo de cálcio

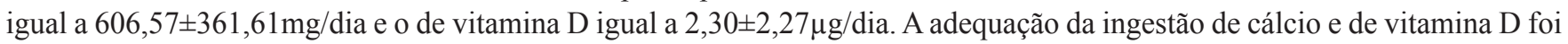
igual a $52,82 \%$ e $12,47 \%$, respectivamente, em que se calculou a média de consumo do nutriente pelos participantes do estudo, dividido pelo valor recomendado, multiplicado por 100 para ser expresso em $\%$.

A recomendação de vitamina $\mathrm{D}$ é de $15 \mu \mathrm{g}$ diários. Dos participantes, $89,5 \%(\mathrm{n}=895)$ apresentaram consumo de vitamina D abaixo da recomendação, da mesma forma que $86,6 \%(n=866)$ dos participantes apresentaram consumo de cálcio inferior à recomendação, que é de $1.000 \mathrm{mg}$ diários, entre 20 e 59 anos, para ambos os sexos, sendo de $1.200 \mathrm{mg}$ diários para o sexo feminino a partir dos 50 anos de idade.

A Tabela I apresenta o consumo de cálcio e de vitamina D de acordo com o sexo, demonstrando que o masculino apresentou ingestão, significativamente, maior de cálcio em comparação ao feminino, enquanto o consumo de vitamina D não apresentou diferença significativa entre os sexos $(\mathrm{p}=0,307)$. Quando avaliada a frequência relativa de participantes que apresentaram ingestão igual ou maior à recomendação, foi observado que $21,9 \%(n=52)$ dos homens e $10,7 \%(n=82)$ das mulheres apresentaram ingestão adequada de cálcio, $8,0 \%(\mathrm{n}=19)$ dos homens e $11,3 \%(\mathrm{n}=86)$ das mulheres apresentaram ingestão adequada de vitamina $\mathrm{D}(\mathrm{p}=0,001)$. 
Tabela I - Ingestão de cálcio e de vitamina D de acordo com o sexo dos participantes. Rio Grande do Sul, 2015-2016.

\begin{tabular}{llll}
\hline Variáveis & $\begin{array}{c}\text { Masculino } \\
(\mathbf{n = 2 3 7 )} \\
\mathbf{m e ́ d i a} \pm \mathbf{D P}\end{array}$ & $\begin{array}{c}\text { Feminino } \\
(\mathbf{n = 7 6 3 )} \\
\mathbf{m e ́ d i a}^{*} \pm \mathbf{D P}\end{array}$ & Valor $\mathbf{p}$ \\
\hline Cálcio $(\mathbf{m g})$ & $705,31 \pm 418,70$ & $575,87 \pm 336,36$ & $<0,001^{1}$ \\
Vitamina D $(\boldsymbol{\mu g})$ & $2,43 \pm 2,31$ & $2,25 \pm 2,25$ & $0,307^{1}$ \\
Ingestão adequada de cálcio & $52(21,9 \%)$ & $82(10,7 \%)$ & $<0,001^{2}$ \\
Ingestão adequada de vitamina D & $19(8,0 \%)$ & $86(11,3 \%)$ & $0,153^{2}$ \\
\hline
\end{tabular}

*Dados apresentados como média \pm desvio padrão.1: Teste $t$ de Student para amostras independentes. 2: Teste qui-quadrado.

Os participantes foram agrupados em quartis de acordo com a faixa etária para comparação da ingestão de cálcio e vitamina $\mathrm{D}$, sendo observada maior ingestão desses micronutrientes no grupo de participantes com idade entre 41 e 50 anos, conforme demonstrado na Tabela II. Os grupos diferiram significativamente quanto ao consumo de vitamina D, sendo observada uma tendência à diferença significativa no consumo de cálcio de acordo com a idade dos participantes $(\mathrm{p}=0,063)$.

Tabela II - Ingestão de cálcio e de vitamina D de acordo com os quartis de idade. Rio Grande do Sul, 2015-2016.

\begin{tabular}{lcc}
\hline Variáveis & Cálcio $(\mathbf{m g})$ & $\begin{array}{c}\text { Vitamina D }(\boldsymbol{\mu g}) \\
\text { média }^{*} \mathbf{D P} \mathbf{P}^{*}\end{array}$ \\
\hline$\leq \mathbf{3 0}$ anos $(\mathbf{n}=\mathbf{8 5 1})$ & $594,71 \pm 363,40$ & $2,17 \pm 2,01^{\mathrm{a}}$ \\
$\mathbf{3 1} \mathbf{- 4 0}$ anos $(\mathbf{n}=\mathbf{1 0 7})$ & $659,78 \pm 357,11$ & $2,87 \pm 3,01^{\mathrm{b}, \mathrm{c}}$ \\
$\mathbf{4 1} \mathbf{- 5 0}$ anos $(\mathbf{n}=\mathbf{3 0})$ & $731,77 \pm 274,61$ & $3,86 \pm 2,84^{\mathrm{c}}$ \\
$\mathbf{\mathbf { 5 0 }}$ anos $(\mathbf{n}=\mathbf{7})$ & $698,98 \pm 421,52$ & $1,82 \pm 2,65^{\mathrm{a}, \mathrm{b}, \mathrm{c}}$ \\
Valor $\mathbf{p}$ & 0,063 & $<0,001$ \\
\hline
\end{tabular}

*Dados apresentados como média \pm desvio padrão. Teste Anova seguido de Tukey. Letras sobrescritas distintas indicam diferença significativa entre os grupos e correspondem a $(\mathrm{p}<0,05)$.

\section{DISCUSSÃO}

Mais da metade dos pacientes avaliados no presente estudo apresentaram inadequação na ingestão de cálcio e vitamina $\mathrm{D}$ em relação aos valores diários recomendados pelas DRIs, sugerindo a necessidade de conscientização dessa população sobre a importância da ingestão adequada desses micronutrientes, visando a promoção e prevenção da saúde no que diz respeito a prováveis complicações, a exemplo da osteoporose, que vem sendo considerada um problema de saúde pública mundial ${ }^{(14,16)}$.

Uma revisão sistemática de metanálise indicou alta prevalência de inadequação em nutrientes na população adulta, dentre eles, cálcio e vitamina $\mathrm{D}^{(11)}$. Atualmente, há evidências de que a vitamina $\mathrm{D}$ desempenha diversas funções além do metabolismo do cálcio ${ }^{(16-19)}$, sendo um dos minerais mais importantes para a formação e reabsorção óssea, o qual é responsável pela "dureza" e proteção de tecidos moles ${ }^{(10,20)}$, além de aumentar a mobilização de cálcio no esqueleto, resultando em osso porótico, a partir do hiperparatireoidismo secundário ${ }^{(21)}$.

A osteoporose é uma doença óssea silenciosa ${ }^{(22)}$, definida por uma desordem esquelética ${ }^{(23)}$, podendo acometer $17 \%$ das mulheres e $7 \%$ dos homens ${ }^{(24)}$, que ocorre devido a diversos fatores, caracterizando-se pela perda de massa óssea e deterioração dos ossos, tornando-os mais frágeis e suscetíveis às fraturas ${ }^{(14,22)}$. $\mathrm{O}$ aumento do consumo de alimentos fontes de cálcio e vitamina D surtiu efeito positivo na perda da densidade mineral óssea $(\mathrm{DMO})^{(25,26)}$. Existem recomendações que indicam a ingestão adequada desses nutrientes associada ao mineral fósforo após os 50 anos de idade, representando melhora na DMO e reduzindo risco de osteoporose por fraturas em 45\%, o que configura um benefício para a saúde óssea ${ }^{(27-30)}$.

Diversos achados revelaram baixos níveis de ingestão, tanto da vitamina D quanto de cálcio, através de estudos da utilização de recordatórios alimentares ${ }^{(16,19,31-36)}$, assim como outros estudos, quando estratificado por faixa etária, também demonstraram um consumo médio de cálcio abaixo do preconizado em indivíduos com idades entre 19 e 65 anos ${ }^{(16,19,31-37)}$, corroborando os dados do presente estudo, sabendo que o recomendado é de 1.000 a $1.200 \mathrm{mg}$ por dia, dependendo da faixa etária e sexo. Em vista dos dados descritos e encontrados, essa situação torna-se preocupante, uma vez que indicações da literatura atual demonstram o efeito preventivo do cálcio para diversas doenças ${ }^{(32)}$.

Estudos de coorte ${ }^{(27,38)}$, que avaliaram o consumo de cálcio através de questionários de frequência alimentar, apresentaram uma média de ingestão de cálcio de $792 \pm 428 \mathrm{mg}$ por dia para ambos os sexos, e de $572 \pm 150 \mathrm{mg}$ para o feminino, semelhante ao valor encontrado no presente estudo para este sexo, o que pode se caracterizar como um risco para o desenvolvimento de doenças ósseas, pela ingestão de teores insatisfatórios do mineral em comparação às recomendações atuais ${ }^{(39)}$.

Outro estudo realizado com 233 indivíduos, de ambos os sexos, verificou que, em todas as faixas etárias avaliadas, nenhum participante atingiu as recomendações diárias de cálcio segundo a Estimated Average Requirements (EAR) ${ }^{(13)}$, não apresentando diferença significativa entre os $\operatorname{sexos}^{(40)}$. Outra análise, com objetivo de estudar o consumo alimentar via registros alimentares, 
avaliou 34.003 indivíduos com idade igual ou superior a 10 anos, agrupando-os por faixas etárias (10-18 anos, 19-59 anos e $\geq 60$ anos), e apresentou resultados de ingestão de cálcio e vitamina D diários de $511 \mathrm{mg}$ e $3,18 \mu \mathrm{g}$, respectivamente, na categoria entre 19-59 anos de ambos os $\operatorname{sexos}^{(35)}$. No presente estudo, a média de ingestão de cálcio e vitamina $\mathrm{D}$ variou entre as faixas etárias estudadas, demonstrando valores inferiores ao recomendado para todas as categorias. Esses valores são sugestivos de possíveis consequências futuras, como, falha na manutenção da homeostase do cálcio, o qual se torna vital para o bom funcionamento do sistema nervoso, crescimento e para manutenção da densidade óssea ${ }^{(10)}$.

Em um estudo transversal, encontrou-se $69,2 \%$ de inadequação em mulheres com mais de 26 anos, entretanto, entre as mulheres com menos de 26 anos, foi constatado um índice de 88,2\%(31). Quando comparadas entre as regiões brasileiras, também se constataram elevadas prevalências de inadequação na ingestão de cálcio e vitamina D pela população adulta, entre 20 e 59 anos, onde a média de inadequação de cálcio na população foi de $84 \%$ e $92 \%$ entre homens e mulheres, respectivamente, e $99,6 \%$ de vitamina D em ambos os sexos ${ }^{(1)}$. Resultados semelhante ao estudo atual, que encontrou $78,1 \%$ de inadequação de cálcio entre homens e $89,3 \%$ em mulheres, e $92 \%$ de inadequação de vitamina D em homens e $88,7 \%$ em mulheres entre 20 a 59 anos. Um dos achados para a justificativa da menor inadequação no consumo de cálcio por homens observado em outro estudo foi o aumento do consumo de energia em comparação às mulheres ${ }^{(1)}$.

Em relação ao consumo de vitamina D em demais estudos realizados com mulheres, foi encontrada uma ingestão de 3,08 $\mu \mathrm{g}$ por $\operatorname{dia}^{(40)}$ e $1,2 \mu \mathrm{g}$ por dia ${ }^{(17)}$. Quanto à diferenciação entre os sexos, outro estudo apresentou valores médios de ingestão de $2,18 \pm 2,31 \mu \mathrm{g}$ e $1,72 \pm 1,56 \mu \mathrm{g}$ por dia na faixa etária dos 31 a 50 anos, para homens e mulheres, respectivamente, e $2,70 \pm 1,84 \mu \mathrm{g}$ e $2,98 \pm 2,12 \mu \mathrm{g}$ por dia para homens e mulheres, respectivamente, na faixa etária dos 51 a 70 anos ${ }^{(35)}$, mostrando semelhança aos achados do presente estudo, pois, para homens, os valores médios permaneceram em $2,43 \pm 2,31 \mu$ g por dia e, para mulheres, $2,25 \pm 2,25 \mu \mathrm{g}$ por dia na faixa etária de 20 a 59 anos, provavelmente oriundos de inadequação ao baixo consumo de produtos ricos ou enriquecidos e baixa exposição solar ${ }^{(16,41)}$.

As limitações do presente estudo estão vinculadas às informações coletadas nos recordatórios alimentares de 24 horas, o qual expressou o consumo atual, e não habitual, pois esse foi de apenas um dia, já que era o que estava disponível nos prontuários avaliados.

\section{CONCLUSÃO}

Por meio deste estudo, conclui-se inadequação quanto à ingestão de cálcio e vitamina D na população estudada, representando alto risco para o desenvolvimento de doenças ósseas, uma vez que a grande maioria dos participantes ingere quantidades significativamente inferiores às recomendações difundidas atualmente.

\section{CONFLITOS DE INTERESSE}

Autores declaram que não há conflitos de interesse na publicação deste artigo.

\section{REFERÊNCIAS}

1. Leite SC, Baratto I, Silva R. Consumo de cálcio e risco de osteoporose em uma população de idosos. RBONE. 2014;8(48):165-74.

2. Lima AC. A utilização de cálcio e vitamina D na prevenção e no tratamento da osteoporose: Uma revisão de literatura. REBES. 2014;4(1):50-6.

3. Araujo MC, Bezerra IL, Barbosa FS, Junger WL, Yokoo EM, Pereira RA, et al. Consumo de macronutrientes e ingestão inadequada de micronutrientes em adultos. Revista de Saúde Pública, São Paulo. 2013;47(Supl 1):177s-89s.

4. Maeda SS, Lazaretti-Castro M. An overview on the treatment of postmenopausal osteoporosis. Arq Bras Endocrinol Metab. 2014;58(2):162-71.

5. Teixeira TM, Costa CL. Papel da vitamina D no lúpus eritematoso sistêmico. Rev Nutr. 2012;25(4):531-8.

6. Institute of Medicine. Dietary reference intakes for calcium and vitamin D. Washington: The National Academies Press; 2010 .

7. Inda AJ Filho, Melamed ML. Vitamina D e doença renal. O que nós sabemos e o que nós não sabemos. J Bras Nefrol. 2013;35(4):323-31.

8. Mozos I, Marginean O. Links between vitamin D deficiency and cardiovascular diseases. Biomed Res Int. 2015;2015:109275.

9. Lichtenstein A, Ferreira MJ, Sales MM, Aguiar FB, Fonseca LAM, Sumita NM, et al. Vitamin D: non-skeletal actions and rational use. Rev Assoc Med Bras. 2013;59(5):495-506. 
10. França NAG, Martini LA. Funções plenamente reconhecidas de nutrientes: cálcio. São Paulo: ILSI; 2014.

11. Borg ST, Verlaan S, Hemsworth J, Mijnarends DM, Schols JMGA, Luiking YC, et al. Micronutrient intakes and potential inadequacies of community-dwelling older adults: a systematic review. Br J Nutr. 2015;113(8):1195-206.

12. Leão LS, Cardoso FS. Efeitos do consumo de cálcio na composição corporal e perda de peso em adultos. RBCS. 2014;12(40):69-73.

13. Dietary reference intakes for calcium. Washington: The National Academies Press; 2010.

14. Basile LH. Gestante e necessidade da vitamina D. Int J Nutrology. 2014;7(1):5-13.

15. Bosco SMD, Conde SR, Machado IK. Métodos práticos para cálculo de dietas. Lajeado: Univates; 2007.

16. Ethgen O, Hiligsmann M, Burlet N, Reginster JY. Public health impact and cost-effectiveness of dairy products supplemented with vitamin D in prevention of osteoporotic fractures. Arch Public Health. 2015;73:48.

17. Horta PM, Souza AC Lopes, Santos LC. Dietary quality maintained among overweight brazilian women enrolled in a primary healthcare service. J Health Popul Nutr. 2014;32(4):608-14.

18. Bellan M, Pirisi M, Sainaghia PP. Osteoporose na artrite reumatoide: papel do sistema vitamina D/hormônio paratireóideo. Rev Bras Reumatol. 2015;55(3):256-63.

19. Rafaelli RA, Nomura PR, Figueira FD, Santos ICPF, Silva LFRS, Venturini D. Influência da vitamina D nas doenças endocrinometabólicas. Semina Ciênc Biol Saúde. 2015;36(1 Supl):333-48.

20. Ahmadieh H, Arabi A. Vitamins and bone health: beyond calcium and vitamin D. Nutr Rev. 2011;69(10):584-98.

21. Choi S, Yeum K, Park S, Choi B, Joo N. Dietary calcium and framingham risk score in vitamin d deficient male (KNHANES 2009-2011). Yonsei Med J. 2015;56(3):845-52.

22. Bonucci E. Bone mineralization. Front Biosci (Landmark Ed). 2012;17:100-28.

23. Pludowski P, Holick MF, Pilz S, Wagner CL, Hollis BW, Grant WB et al. Vitamin D effects on musculoskeletal health, immunity, autoimmunity, cardiovascular disease, cancer, fertility, pregnancy, dementia and mortality-A review of recent evidence. Autoimmun Rev. 2013;12(10):976-89.

24. Zhu K, Prince RL. Calcium and bone. Clin Biochem. 2012;45(12):936-42.

25. Souza MPG. Diagnóstico e tratamento da osteoporose. Rev Bras Ortop. 2010;45(3):220-9.

26. Fontes TMP, Araújo LFB, Soares PRG. Osteoporose no climatério I: epidemiologia, definição, rastreio e diagnóstico. Femina. 2012;40(2):109-16.

27. Tai V, Leung W, Grey A, Reid IR, Bolland MJ. Calcium intake and bone mineral density: systematic review and metaanalysis. BMJ. 2015;351:4183.

28. Khan B, Nowson CA, Daly RM, English DR, Hodge AM, Giles GG, et al. Higher dietary calcium intakes are associated with reduced risks of fractures, cardiovascular events, and mortality: a prospective cohort study of older men and women. J Bone Miner Res. 2015;30(10):1758-66.

29. Lee AW, Cho SS. Association between phosphorus intake and bone health in the NHANES population. Nutr J. 2015;14:28.

30. Wadolowska L, Sobas K, Szczepanska JW, Slowinska MA, Czlapka-Matyasik M, Niedzwiedzka E. Dairy products, dietary calcium and bone health: possibility of prevention of osteoporosis in women: the polish experience. Nutrients. 2013;5(7):2684-707.

31. Andrade SAF. Osteoporose: um problema de saúde pública. Unilus Ensino Pesquisa. 2015;12(28):41-6.

32. Domene SMA, Assumpção D, Barros MBA, Luz VG, Fisberg RG. Ingestão de minerais e fitatos: indicadores para o monitoramento de risco nutricional. Vig Sanit Debate. 2014;2(4):69-75.

33. Cardoso AMG, Barcelos RS, Mesenburg MA, Silva CML. Prevalência do consumo de cálcio em universitárias. Rev Acadêmica Medicina UFPEL. 2013;7(1):1-3.

34. Viudes DR, Brecailo MK, Schwabsilva J, Levinske LC, Melhem ARF, Kühl AM. Perfil nutricional e consumo alimentar de pacientes com excesso de peso atendidos por um ambulatório de nutrição. Publ UEPG Ci Biol Saúde. 2014;20(2):115-24.

35. Steiner ML, Azevedo LH, Bonacordi CL, Barros AZ, Strufaldi R, Fernandes CE. Avaliação de consumo alimentar, medidas antropométricas e tempo de menopausa de mulheres na pós-menopausa. Rev Bras Ginecol Obstet. 2015;37(1):16-23. 
36. Murphy MM, Barraj LM, Toth LD, Laura S Harkness LS, Bolster DR. Daily intake of dairy products in Brazil and contributions to nutrient intakes: a cross-sectional study. Public Health Nutr. 2016;19(3):393-400.

37. Ruiz FS, Oliveira AF, Simão ANC, Lozovoy MAB, Alfieri AF, Sandrini F, et al. Associação entre deficiência de vitamina $\mathrm{D}$, adiposidade e exposição solar em participantes do sistema de hipertensão arterial e diabetes melito. Semina Ciênc Biol Saúde. 2014;35(2):103-14.

38. Souza LB, Malta MB, Donato PM, Corrente JE, Carvalhaes MABL, Papini JS. Inadequação de consumo alimentar, antropometria e estilo de vida de universitárias da área de saúde. J Health Sci Inst. 2012;30(4):377-81.

39. Michaëlsson K, Melhus H, Lemming EW, Wolk A, Byberg L. Long term calcium intake and rates of all cause and cardiovascular mortality: community based prospective longitudinal cohort study. BMJ. 2013;346:228.

40. Silva MR, Costa RR, Souza GN, Faria LS, Sampedro GD. Fatores associados ao consumo de leite fluido em comunidades limítrofes rural-urbanas. Rev Med Minas Gerais. 2014;24(Supl 5):S33-S6.

41. Anta RMO, Rodríguez LGR, Lombán BM, Sánchez JMP, Vizuete AA, Sobaler AML. Ingesta de calcio y vitamina D en una muestra representativa de mujeres españolas; problemática específica en menopausia. Nutr Hosp. 2013;28(2):306-13.

\section{Endereço para correspondência:}

Bruna Amanda Martini

Centro Universitário UNIVATES

Rua Avelino Tallini, 171

Bairro: Bairro Universitário

CEP: 95914-014 - Lajeado - RS

E-mail: nutribrunamartini@gmail.com 